\title{
Performance Analysis of IP-Based Multimedia Communication Networks to Support Video Traffic $^{\star}$
}

\author{
Alexander F. Yaroslavtsev ${ }^{1}$, Tae-Jin Lee ${ }^{2}$, Min Young Chung ${ }^{2}$, and \\ Hyunseung $\mathrm{Choo}^{2}$ \\ 1 Institute of Mining, Siberian Branch of the Russian Academy of Science \\ Novosibirsk, Russia +7-3832-170930 \\ yar@misd.nsc.ru \\ 2 School of Information and Communication Engineering, Sungkyunkwan University \\ 440-776, Suwon, Korea +82-31-290-7145 \\ \{tjlee, mychung, choo\}@ece.skku.ac.kr
}

\begin{abstract}
With the rapid growth of the communication equipment performance, it is possible that communication networks, which use TCP/IP, will be able to provide real-time applications such as the broadcasting of a multi-media traffic. These applications are sensitive to transmission delay and its variance, thus estimating QoS is very important. In this paper, we evaluate the performance of an IP-based multimedia network in terms of the end-to-end mean delivery time and the utilization of communication equipments.
\end{abstract}

Keywords: Performance evaluation, video traffic, MPEG encoding.

\section{Introduction}

In order to support multi-media services in IP-based networks, it is important to assure service qualities, e.g., delay, since IP networks inherently provide best effort service. In general, modeling of IP-based networks supporting multi-media services is complex and thus QoS estimation is challenging.

There have been previous works, which investigate transmission of multimedia traffic over IP-networks [1, [2], 3], 4, [5]. Usually simulation methods are used to research such communication networks. But this approach has a series of limitations. There are great difficulties in adequate representation of transmission of multi-media traffic by analytical models. In 6, Yaroslavtsev et al. proposed an analytic method based on queuing network theory to model IP-based high speed communication networks, which is more adequate than conventional analytical models, and has less computational load than simulation models.

* This paper was partially supported by BK21 program. Dr. Choo is the corresponding author. 
In this paper, we simply evaluate probabilistic temporal characteristics of an IP-based multimedia network in terms of average delay of data flows and utilization of equipment. This paper is organized as follows. In Section 2, we discuss related work about performance evaluation of communication networks and modeling of multi-media traffic. And we present the model of the investigated network and the multi-media services as well. Section 3 shows some performance results on the model of the system.

\section{Description of Modeled Communication Network}

In this paper, as a representation of communication networks, we consider the video transmission in an IP network shown in Fig. 11 We have $N_{w}$ clients connected to a video server via a switch. Each client is connected to the switch through Ethernet port and an information server is connected to the switch through fast Ethernet port. To describe the considered network, we use the set of traffic, hardware and software parameters.

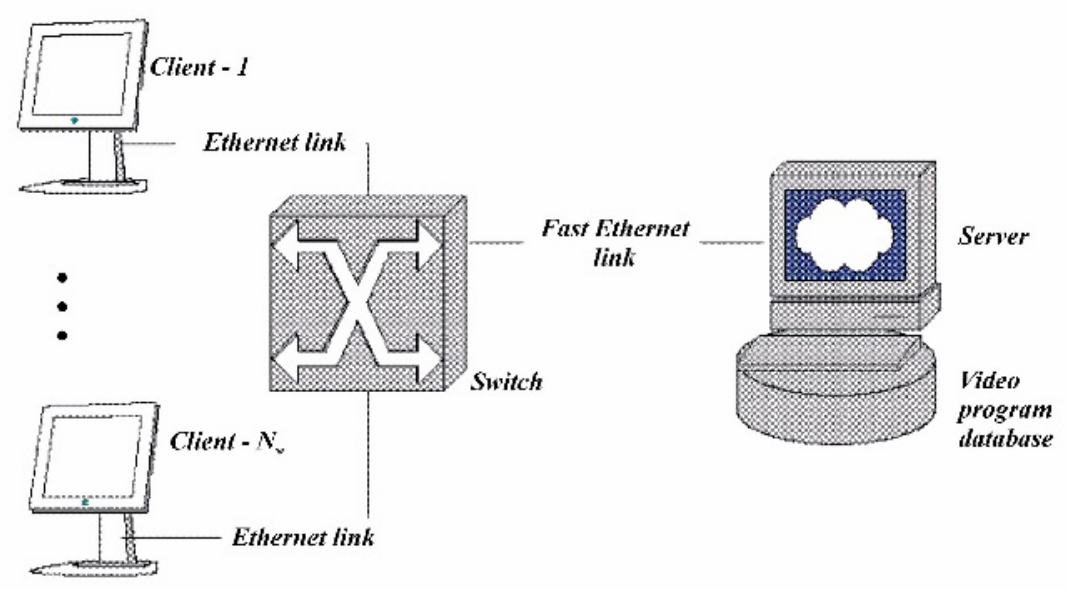

Fig. 1. A structure of IP network, for which QOS was estimated

Traffic parameters describe the characteristics of transferred traffic to the clients. Video stream must be encoded into an MPEG-4 format 7. According to this standard, a digital video stream consists of a sequence of groups of video pictures (GoP). Each GoP consists of the $M / Z$-sequence of video frames (VOP - Video Object Plane). Each GoP or $M / Z$-sequence has fixed length and structure. In GoP three types of video frame are defined: $I$-frame (Intra), $P$-frame (Predictive) and $B$-frame (Bidirectional). In the sequel, we will designate the set of all frame types as $\Omega=\{I, P, B\}$. Each GoP in video stream has one $I$-frame, the first frame in GoP. The parameter $M$ defines the number of frames in groups. The parameter $Z$ is the distance between $P$-frames in GOP. For example, the 
structure of group of a typical digital video stream with parameters $15 / 3$ has the following frames: $I B B P B B P B B P B B P B B$. Values of video traffic parameters are summarized in [6].

Hardware parameters describe the hardware characteristic associated with communication lines (distance, rate, and Bit Error Rate (BER)); performance of computers (server and nodes); rate of the switch (forwarding rate and size of its shared memory). Values of hardware parameters are given in [6].

Software parameters define the characteristics of the network software, which realize the protocol of video data transmission to clients. An end-to-end TCP connection between a client and the server is established. Video frames are encapsulated in TCP segments, and are transmitted along the network to the clients by IP packets. When IP packets are transmitted over the network, they undergo random delays, and they can be received with errors or lost. All these events cause retransmission of appropriate TCP segments and, hence, incur additional delays. Software parameters are in detail illustrated in [6].

\section{Performance Evaluation and Summary}

The proposed model allows to estimate a wide set of performance metrics for video traffic transmission over communication networks, e.g., mean number of IP packets associated with each client in each communication equipment, utilization of each communication equipment, size of required buffer in switch or server, and traffic rate and delay in transmitting each frame type. For illustration, we compute several performance metrics as functions of the number of clients. We have scaled up the traffic parameters so that the traffic rate is equal to $2 \mathrm{Mbps}$ for each client.

To evaluate the performance of the considered network, we define the mean delivery time of $\mathrm{I} / \mathrm{P} / \mathrm{B}$ frames from the server to a client node as the time interval between a new I, P, or B frame generated by the server and a correctly received by the client node. The mean delivery time of each type of frames is shown in Fig. 2. The mean delivery time of I-frame is considerably greater than that of $\mathrm{B}(\mathrm{P})$-frame because the size of I-frame is greatly higher than that of other types. Most of these delivery times is caused by delays in the Ethernet link for less than 20 clients. The figure shows that there is considerable amount of remaining time for frames to be delivered to clients. Let $\lambda^{V O P}$ denote the generating rate of VOP stream for each client. This remaining time is equal to $1 / \lambda^{V O P}=0.04 \mathrm{sec}$. And, quality of broadcasting video stream is guaranteed in the communication network for less number of clients than 20. For more than 25 clients, these delivery times are mostly incurred from delays in fast Ethernet links and Server, and the metric shows exponential growth.

Fig. 3 shows the utilization of equipments such as fast ethernet link, switching fabric, and server in Fig. 1. The server and the fast Ethernet link have more considerable load than the other communication equipments. We see that these coefficients of utilization exhibit unreasonable level for the client number larger than 25. These equipments are bottlenecks and they can reduce QoS level considerably. 


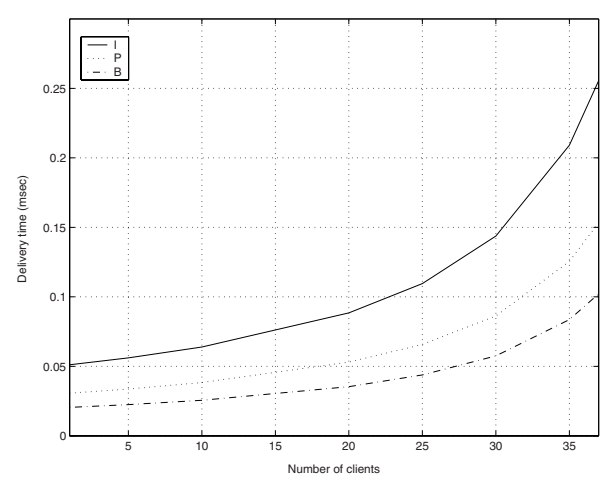

Fig. 2. Mean delivery time of $\omega$-frames from the server to the first client node

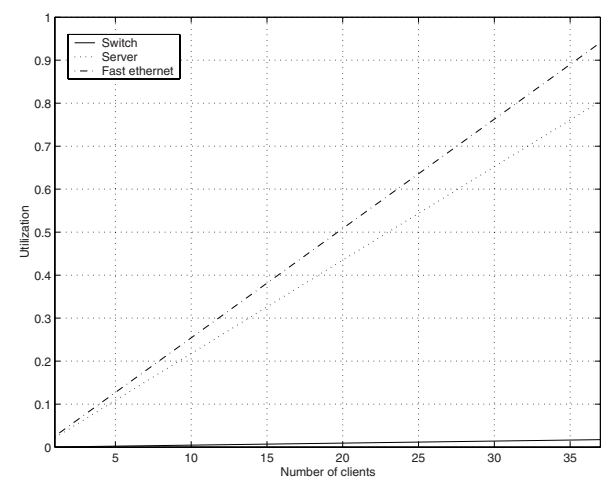

Fig. 3. The utilization of various communication equipment

The results show that the modeled communication network, represented in Fig. 1. sufficiently transmits video traffic and provides simultaneous broadcasting of a digital video to 20-25 clients without significant loss of QoS. Its characteristics is almost linear until the number of clients increases to more than 25. For 35-40 client nodes, the modeled communication network is shown to be overloaded. Fast Ethernet link and server are bottlenecks in the modeled communication network. Thus the proposed modeling method can be used to investigate performance of communication networks with more complex topologies.

\section{References}

1. Zheng, L., and Zhang, L.: Modeling and Performance Analysis for IP Traffic with Multi-Class QoS in VPN. IEEE MILCOM Vol. 1 (2000) 330-334

2. Tian, T., Li, A.H., Wen, J., and Villasenor, J.D.: Priority Dropping in Network Transmission of Scalable Video. Int. Conf. on Image Processing Vol. 3 (2000) 400403

3. Wu, D., Hou, Y.T., Zhang, Y.-Q., and Chao, H.J.: Optimal Mode Selection in Internet Video Communication: an End-to-End Approach. IEEEE ICC Vol. 1 (2000) 264-271.

4. F. Beritelli, G. Ruggeri and G. Schembra: TCP-friendly Transmission of Voice over IP. IEEE ICC Vol. 2 (2002) 1204-1208

5. de Carvalho Klingelfus, A.L. and Godoy Jr., W.: Mathematical Modeling, Performance Analysis and Simulation of Current Ethernet Computer Networks. 5th IEEE Int. Conf. on High Speed Networks and Multimedia Commun. (2002) 380-382

6. Yaroslavtsev, A.F., Lee, T.-J., Chung, M.Y., and Choo, H.: Performance Analysis of IP-Based Multimedia Communication Networks to Support Video Traffic.

TR-ECE03-001 Sungkyunkwan University (2003)

7. Overview of the MPEG-4 standard. ISO/IEC JTC1/SC29/WG11 2459 (1998) 